\title{
MULTIPLIER EXTENSIONS OTHER THAN THE MACKEY EXTENSION
}

\author{
LARRY BAGGETT
}

\begin{abstract}
The set of all multiplier extensions to the whole group $G$ of a representation of a normal subgroup $N$ is completely described, and necessary and sufficient conditions are given for equivalence of two such extensions.
\end{abstract}

I. Introduction. In his celebrated Acta paper of 1958, G. W. Mackey describes a method for constructing irreducible unitary representations of a group extension. Indeed, in the presence of some extra assumptions, all the irreducible representations of such an extension are constructed via this method. One of the integral parts of the procedure is in the extending of a representation of one subgroup to a larger subgroup. There are, in general, other ways of extending the representation than the one Mackey chose, and it seems likely that some more information might be forthcoming by utilizing these "other" extensions. It is our purpose here to describe completely the set of all such extensions and to give necessary and sufficient conditions for two such extensions to be equivalent. Let us establish some notation and state the relevant Mackey results.

Suppose $N$ is a closed normal subgroup of a separable locally compact group $G$. If $L$ is any representation of $N$ and if $x$ is an element of $G$, let $x \cdot L$ be the representation of $N$ defined by $(x \cdot L)_{n}=L_{\left(x^{-1} n x\right)}$. Then, since $L$ being equivalent to $L^{\prime}$ implies that $x \cdot L$ is equivalent to $x \cdot L^{\prime}$, the dual space $\hat{N}$ of $N$ becomes a $G$-space. Actually it is convenient to think of $\hat{N}$ as a $G / N$-space as well. The group $G / N$ does act on $\hat{N}$ because $x \cdot L$ is always equivalent to $L$ if $x$ belongs to $N$.

Now let $L$ be an irreducible unitary representation of $N$ for which $x \cdot L$ is equivalent to $L$ for all $x$ in $G$.

THEOREM 1.1 (MACKEY). There exists a multiplier representation $M$ of $G$ with multiplier $\tau$ such that:

(i) $M$ extends $L$, i.e., the space of $M$ is the space of $L$ and $\left.M\right|_{N}$ is $L$.

(ii) The multiplier $\tau$ is the inflation to $G \times G$ of a multiplier $\omega$ on $G / N \times G / N$.

(iii) The representation $M$ is unique in the sense that, if $M^{\prime}$ is another multiplier representation of $G$ which extends $L$ and whose multiplier $\tau^{\prime}$ is the inflation of a multiplier $\omega^{\prime}$ on $G / N \times G / N$, then $\tau$ and $\tau^{\prime}$ are cohomologous and $M$ and $M^{\prime}$ are cohomologous representations. (Two multiplier representations $M$ and $M^{\prime}$ are cohomologous if there exists a unitary operator $v$ from the space of $M$ onto the

Received by the editors March 1, 1975.

AMS (MOS) subject classifications (1970). Primary 22D10, 22D12.

C American Mathematical Society 1976 
space of $M^{\prime}$ and a Borel function $\beta$ from $G$ into the circle group $T^{1}$ so that $M_{x}=\beta(x) v^{-1} M_{x}^{\prime} v$ for all $x$.)

It is condition (iii) here which is slightly misleading. The point is that, if the multiplier $\tau^{\prime}$ is not required to be an inflation, then there are of ten many noncohomologous extensions of $L$.

The proof to Mackey's theorem has its difficulty only in the measure theory. Intuitively we see that we must define $M_{x}$ to be an operator on the space of $L$ so that

$$
M_{x}^{-1} L_{n} M_{x}=L_{\left(x^{-1} n x\right)} \text { for all } n .
$$

Such an operator exists and is unique up to scalar operators because $L$ is irreducible. The difficulty then is in finding a measurable selection of these operators.

Finally, let us state another theorem of Mackey's which indicates how the "Little Group" gets into the picture. We shall always write $\pi$ for the projection of $G$ onto $G / N$.

THEOREM 1.2 (MACKeY). Let $N, G, L, M, \tau$ and $\omega$ be as in the above discussion. If $R$ is an irreducible representation of $G$ whose restriction to $N$ is a multiple of $L$, then there exists a $\bar{\omega}$-representation $W$ of $G / N$ such that $R$ is equivalent to $M \otimes(W \cdot \pi)$.

One wonders how other extensions of $L$ could be combined to construct representations of $G$. Perhaps the tensor product of some extension $M^{\prime}$ of $L$ with a $\overline{\tau^{\prime}}$-representation would yield a unitary representation of $G$ whose restriction to $N$ is concentrated on, for example, a properly ergodic measure class. We have not yet found such an example, but the possibilities clearly exist. In §III we shall apply our theorem to some special groups and representations.

II. Multiplier extensions. Suppose $N$ is a closed normal subgroup of a separable locally compact group $G$, and let $L$ be an irreducible unitary representation of $N$. Assume further that for all $x$ in $G$ we have $x \cdot L$ is equivalent to $L$. We shall compute in this section all the multiplier extensions of $L$ to $G$, and we shall give necessary and sufficient conditions for two such extensions to be cohomologous. (See Theorem 1.1(iii) for the definition of "cohomologous representations".) Denote by $M$ a fixed Mackey extension of $L$ with multiplier $\tau$, and recall that for all $n$ in $N$ and $x$ in $G$, we have

$$
M_{x}^{-1} L_{n} M_{x}=L_{\left(x^{-1} n x\right)}=(x \cdot L)_{n} .
$$

Now let $\Gamma(L)$ denote the set of all characters $\varphi$ on $N$ for which $\varphi L$ is equivalent to $L$. If $L$ itself is a character, then $\Gamma(L)$ is trivial, the trivial character only, but in general this set is not trivial. Indeed if $L$ is induced from a normal subgroup $N^{\prime}$ of $N$, say $L=U^{L^{\prime}}$, then for any character $\varphi$ of $N / N^{\prime}$, we have $\varphi L=\varphi U^{L^{\prime}}$ which is equivalent to $U\left(\left.\varphi\right|_{N^{\prime}} L^{\prime}\right)$ which is $U^{L^{\prime}}$ which is $L$. So in this case $\Gamma(L)$ contains at least the entire character group of $N / N^{\prime}$. Always $\Gamma(L)$ is a subgroup of the character group $\Gamma(N)$ of $N$, but we shall see in the next section that it need not be closed.

Proposirion 2.1. The set $\Gamma(L)$ is a Borel subgroup of $\Gamma(N)$ and it is invariant under the action of $G$. 
Proof. We have already remarked that $\Gamma(L)$ is a subgroup. If $\mathcal{Q}$ denotes the unitary group on the space of $L$, equipped with the strong operator topology, then the product $\Gamma(N) \times U$ is a Polish topological group. For each element $n$ of $N$, the two mappings $(\varphi, v) \rightarrow \varphi(n) L_{n}$ and $(\varphi, v) \rightarrow v^{-1} L_{n} v$ are continuous from $\Gamma(N) \times \mathcal{U}$ into $थ$. Hence the set of all pairs $(\varphi, v)$ for which $\varphi(n) L_{n}$ $=v^{-1} L_{n} v$ for all $n$ is closed, and it is obviously a subgroup of $\Gamma(N) \times \mathcal{Q}$. The projection of this closed subgroup onto its first coordinate is a continuous homomorphism of this group into a separable locally compact group. Therefore its range, which is clearly $\Gamma(L)$, is at least a Borel subgroup of that group. Hence $\Gamma(L)$ is a Borel subgroup of $\Gamma(N)$.

Finally, if $x$ belongs to $G, \varphi$ belongs to $\Gamma(L)$, and $v$ is an operator for which $\varphi(n) L_{n}=v^{-1} L_{n} v$ for all $n$, then

$$
\begin{aligned}
(x \cdot \varphi)(n) L_{n} & =\varphi\left(x^{-1} n x\right) L_{\left(x x^{-1} n x x^{-1}\right)}=\varphi\left(x^{-1} n x\right) M_{x} L_{\left(x^{-1} n x\right)} M_{x}^{-1} \\
& =M_{x} v^{-1} L_{\left(x^{-1} n x\right)} v M_{x}^{-1}=\left[M_{x} v M_{x}^{-1}\right]^{-1} L_{n}\left[M_{x} v M_{x}^{-1}\right]
\end{aligned}
$$

for all $n$, which shows that $(x \cdot \phi) L$ is equivalent to $L$ and, in fact, that $M_{x} v M_{x}^{-1}$ effects the equivalence between these two representations. In particular, $\Gamma(L)$ is invariant which completes the proof.

Now there is, up to scalar multiples, a unique multiplier representation $\varphi \rightarrow U_{\varphi}$ of $\Gamma(L)$ satisfying

$$
U_{\varphi}^{-1} L_{n} U_{\varphi}=\varphi(n) L_{n} \text { for all } n .
$$

We have seen too, in the course of the last proof, that $U_{(x \cdot \varphi)}$ is a scalar multiple $\delta(x, \varphi)$ of the operator $M_{x} U_{\varphi} M_{x}^{-1}$. Let us denote the multiplier associated with $U$ by $\varepsilon$.

Finally let $\mathscr{F}(L)$ denote the set of all Borel functions $f$ from $G / N$ into $\Gamma(L)$ which satisfy $f(h k)=f(h)(h \cdot f(k))$. These are 1-cocycles of $G / N$ with coefficients in $\Gamma(L)$ and relative to the action of $G / N$ on $\Gamma(L)$. The 1coboundaries are the functions $h \rightarrow(h \cdot \varphi) \bar{\Phi}$ where $\varphi$ is some element of $\Gamma(L)$. It is in fact the group $H^{1}(G / N, \Gamma(L))$ which describes the distinct extensions of $L$ to $G$.

THEOREM 2.2. For each element $f$ of $\mathscr{F}(L)$ define a mapping $S^{f}$ from $G$ into $\mathcal{Q}$ by $S_{x}^{f}=U_{f(\pi(x))} M_{x}$. Then:

(i) $S^{f}$ is a multiplier extension of $L$.

(ii) If $M^{\prime}$ is a multiplier extension of $L$, then there exists an element $f$ of $\mathscr{F}(L)$ and $a$ Borel function $\lambda$ from $G$ into $T^{1}$ such that $M_{x}^{\prime}=\lambda(x) S_{x}^{f}$ for all $x$. In particular any multiplier extension of $L$ is cohomologous to some $S^{f}$.

(iii) $S^{f}$ is cohomologous to $S^{g}$ if and only if $f=g b$, where $b$ is a coboundary.

ProOF.

$$
\begin{aligned}
S_{x}^{f} S_{y}^{f} & =U_{f(\pi(x))} M_{x} U_{f(\pi(y))} M_{y} \\
& =U_{f(\pi(x))}\left[M_{x} U_{f(\pi(y))} M_{x}^{-1}\right] M_{x} M_{y} \\
& =U_{f(\pi(x))} \bar{\delta}(x, f(\pi(y))) U_{(x \cdot f(\pi(y)))} \tau(x, y) M_{x y} \\
& =\tau(x, y) \bar{\delta}(x, f(\pi(y))) \varepsilon(f(\pi(x)),(x \cdot f(\pi(y)))) U_{(f(\pi(x))(x \cdot f(\pi(y))))} M_{x y} \\
& =\tau^{f}(x, y) S_{x y}^{f}
\end{aligned}
$$


where

$$
\tau^{f}(x, y)=\tau(x, y) \bar{\delta}(x, f(\pi(y))) \varepsilon(f(\pi(x)),(x \cdot f(\pi(y)))) .
$$

Hence $S^{f}$ is a multiplier representation. It obviously extends $L$ since $U_{e}$ is the identity, and we have before us a formula for its multiplier $\tau^{f}$. This proves (i).

Next let $M^{\prime}$ be a multiplier representation of $G$ which extends $L$ and with multiplier $\tau^{\prime}$. Then

$$
\begin{aligned}
M_{x}^{\prime-1} L_{n} M_{x}^{\prime} & =\overline{\tau^{\prime}}\left(x, x^{-1}\right) \tau^{\prime}\left(x^{-1}, n\right) \tau^{\prime}\left(x^{-1} n, x\right) L_{\left(x^{-1} n x\right)} \\
& =\varphi_{x}(n) M_{x}^{-1} L_{n} M_{x}
\end{aligned}
$$

where

$$
\varphi_{x}(n)=\overline{\tau^{\prime}}\left(x, x^{-1}\right) \tau^{\prime}\left(x^{-1}, n\right) \tau^{\prime}\left(x^{-1} n, x\right)
$$

Consequently

$$
M_{x} M_{x}^{\prime-1} L_{n} M_{x}^{\prime} M_{x}^{-1}=\varphi_{x}(n) L_{n} \text { for all } n \text {. }
$$

It follows that $\varphi_{x}$ is a character of $N$, and from this last equation we see that $\varphi_{x}$ belongs to $\Gamma(L)$. Furthermore the operator $M_{x}^{\prime} M_{x}^{-1}$ must be a multiple $\lambda(x)$ of the operator $U_{\left(\varphi_{x}\right)}$. Since $M, M^{\prime}$ and $\varphi$ are all Borel functions of $x$, it follows that $\lambda$ must be Borel as well. We have that $M_{x}^{\prime}=\lambda(x) U_{\left(\varphi_{x}\right)} M_{x}$ and (ii) will be proved if we can show that $x \rightarrow \varphi_{x}$ belongs to $\mathscr{F}(L)$. This will follow if we verify the following two facts about this function. $\varphi_{x n}=\varphi_{x}$ for all $x$ in $G$ and $n$ in $N$, and $\varphi_{x y}=\varphi_{x}\left(x \cdot\left(\varphi_{y}\right)\right)$ for all $x$ and $y$ in $G$. Both of these facts are obtained, with persistence, from the cocycle identity for 2-cocycles, and from the definition of $\varphi_{x}$ in terms of the multiplier $\tau^{\prime}$. Note too that we can calculate the function $f$ defining $S^{f}$ from the multiplier $\tau^{f}$. Thus

$$
[f(\pi(x))](n)=\overline{\tau^{f}}\left(x, x^{-1}\right) \tau^{f}\left(x^{-1}, n\right) \tau^{f}\left(x^{-1} n, x\right) .
$$

Now let us prove (iii). Suppose $S^{f}$ is cohomologous with $S^{g}$, i.e., there exists a unitary operator $v$ and a Borel function $\beta$ from $G$ into $T^{1}$ so that

$$
S_{x}^{f}=\beta(x) v^{-1} S_{x}^{g} v \text { for all } x .
$$

Then, computing the multipliers for these representations, we find that

$$
\tau^{f}(x, y)=\tau^{g}(x, y) \beta(x) \beta(y) \bar{\beta}(x y) .
$$

Since both $S^{f}$ and $S^{g}$ restrict to the ordinary representation $L$ on $N$, their multipliers are trivial there, and $\left.\beta\right|_{N}$ must be a character of $N$. We have also, by restricting to $N$, that

$$
\beta(n) L_{n}=v L_{n} v^{-1} \text { for all } n
$$

which shows that $\left.\beta\right|_{N}$ belongs to $\Gamma(L)$. Since we have seen how to compute $f$ from the multiplier $\tau^{f}$, we calculate and find that 


$$
f(h)=\left.g(h)\left(\left.h \cdot \beta\right|_{N}\right) \bar{\beta}\right|_{N}
$$

which proves half of (iii).

Finally, if $\varphi$ is an element of $\Gamma(L)$ and $f$ and $g$ are elements of $\mathscr{F}(L)$ which are related as in (iii) by the coboundary $h \rightarrow(h \cdot \varphi) \bar{\varphi}$, let $\beta$ be a Borel extension of $\varphi$ to all of $G$ and consider the multiplier representation $M^{\prime}$ defined on $G$ by

$$
M_{x}^{\prime}=\bar{\beta}(x) U_{\varphi}^{-1} S_{x}^{f} U_{\varphi}^{\prime} .
$$

In the first place $M^{\prime}$ is cohomologous with $S^{f}$ by its very definition. In the second place $M^{\prime}$ restricts to $L$ on $N$, and so by part (ii) we have that $M_{x}^{\prime}=\lambda(x) S_{x}^{f^{\prime}}$ for some $f^{\prime}$ in $\mathscr{F}(L)$. Of course we can compute $f^{\prime}$ by using the multiplier for $M^{\prime}$, and we find that $f^{\prime}=g$. Therefore $S^{f}$ is cohomologous with $S^{g}$ as desired.

There is another result which has some analogy with Theorem 1.2.

THEOREM 2.3. Let $\sigma$ be a multiplier on $G \times G$ which is identically one on $N \times N$. Suppose $S$ is an extension of $L$ and that $S$ is a $\sigma$-representation of $G$. If $R$ is any irreducible $\sigma$-representation of $G$ whose restriction to $N$ is a multiple of $L$, then there exists an irreducible unitary representation $W$ of $G / N$ such that $R$ is equivalent to $S \otimes(W \cdot \pi)$.

The proof to this theorem follows from the ordinary Mackey analysis but applied to the group $G^{\sigma}$ with normal subgroup $N^{\sigma}$. We shall omit the easy details.

Theorem 2.3 is important, if the non-Mackey extensions are to play a useful role, for it again reduces the analysis to the "Little Group" except that here the fundamental extension is more complicated to construct.

III. Examples. The question can surely be raised as to whether there are ever any non-Mackey extensions of $L$. The answer to that question is in the affirmative, but the question of whether any of these "other" extensions can be exploited in the way that the Mackey extension has been is still unresolved.

Let $N$ and $K$ be separable locally compact groups, and let $G$ be the direct product $N \times K$. Let $L$ be an irreducible unitary representation of $N$ and let $\Gamma(L)$ be as in $\S I I$. Then $\mathscr{F}(L)$ consists of the Borel homomorphisms of $K$ into $\Gamma(L)$. Furthermore, the action of $K$ on $\Gamma(L)$ being trivial, we see that the trivial homomorphism of $K$ into $\Gamma(L)$ is the only coboundary. So if $\Gamma(L)$ is nontrivial, and if there exist nontrivial homomorphisms of $K$ into $\Gamma(L)$, then there will exist non-Mackey extensions of $L$. (Of course the Mackey extension is the one corresponding to the coboundaries.)

The simplest example of this is when $N$ is the symmetric group $S_{3}$ and $K$ is the two-element group $Z_{2}$. Let $L$ be the irreducible 2-dimensional representation of $N$, in which case $\Gamma(L)=\Gamma(N)$ which is isomorphic with $Z_{2}$. There are two distinct homomorphisms of $K$ into $\Gamma(L)$ in this instance, and so there is a non-Mackey extension of $L$ to the entire 12-element group. Naturally in the case of a finite group there will be no new information deriving from this other extension, but it does at least exist.

For a more complicated and tantalizing example, let $N$ be the semidirect 
product $C Z$ of the complex plane $C$ with the group $Z$ of integers, where an integer $k$ acts on a complex number $\lambda$ by $k(\lambda)=e^{i k} \lambda$. Let $L$ be the irreducible unitary representation of $N$ acting in $L^{2}\left(T^{1}\right)$ and defined by

$$
\left[L_{(c, k)}(f)\right](\lambda)=e^{i(i m(\bar{c} \lambda))} f\left(e^{-i k} \lambda\right) .
$$

What is $\Gamma(L)$ ? We observe that $\left.L\right|_{Z}$ is a direct sum of the characters $\left[\varphi_{m}\right]$ of $Z$, where $\varphi_{m}(k)=e^{i k m}$. (The powers of $\lambda$ are eigenvectors for $Z$.) Therefore, in order for a character $\varphi$ of $N$, a character of $Z$ lifted to $N$, to belong to $\Gamma(L)$, it is necessary that $\varphi$ be one of the $\left[\varphi_{m}\right]$. Conversely each $\varphi_{m}$ does belong to $\Gamma(L)$ for the operator $U_{\left(\varphi_{m}\right)}$ defined on $L^{2}\left(T^{1}\right)$ by

$$
\left[U_{\left(\varphi_{m}\right)}(f)\right](\lambda)=\lambda^{-m} f(\lambda)
$$

effects an equivalence between $\varphi_{m} L$ and $L$.

$\Gamma(N)$ is the entire torus $T^{1}$, so that we have an example where $\Gamma(L)$ is not closed.

What group $K$ to use, or more generally how to realize $N$ as a normal subgroup of some group $G$, is not at all clear. It is $N$ itself whose representations are not yet completely describable, and it is possible to speculate about how to discover new concrete representations of $N$. For example, suppose one considers $G=N \times N$. Construct a non-Mackey extension $S^{f}$ of $L$, tensor with a $\tau^{f}$-representation of $G$, and then restrict to the diagonal. What kinds of representations of $N$ can be constructed in this way?

In conclusion we remark that this kind of game can be played with any group $N$. Some warnings are perhaps appropriate: There is nothing at all to this if $N$ is abelian since $\Gamma(L)$ is then always trivial and there are no nonMackey extensions. Unfortunately the most intriguing cases are for groups $N$ which are themselves poorly understood. Finally, there are occasions when $\Gamma(L)$ is large, $\mathscr{F}(L)$ is large, but unhappily each $f$ in $\mathscr{F}(L)$ is a coboundary. An example of this occurs in the group $N^{\sigma}$, where $N$ is the semidirect product mentioned above and $\sigma$ is a nontrivial multiplier on $N \times N$.

\section{BIBLIOGRAPHY}

1. L. Auslander and C. C. Moore, Unitary representations of solvable Lie groups, Mem. Amer. Math. Soc. No. 62 (1966), 1-199. MR 34 \#723.

2. G. W. Mackey, Unitary representations of group extensions. I, Acta Math. 99 (1958), 265-311. MR 20 \#489.

Department of Mathematics, University of Colorado, Boulder, Colorado 80302 